\title{
Chirp rate and instantaneous frequency estimation: application to recursive vertical synchrosqueezing
}

\author{
Dominique Fourer, François Auger, Krzysztof Czarnecki, Sylvain Meignen, and Patrick Flandrin
}

\begin{abstract}
This letter introduces new chirp rate and instantaneous frequency estimators designed for frequency modulated signals. These estimators are first investigated from a deterministic point of view, then compared together in terms of statistical efficiency. They are also used to design new recursive versions of the vertically synchrosqueezed short-time Fourier transform, using a previously published method [1]. This study paves the way to the real-time computation of a time-frequency representation which is both invertible and sharply localized in frequency.
\end{abstract}

Index Terms - time-frequency analysis, synchrosqueezing, reassignment, chirp rate and instantaneous frequency estimation.

\section{INTRODUCTION}

$\mathbf{N}$ ON-stationary signal analysis is a challenging task and usually requires sophisticated tools. To this end, the well-known Short-Time Fourier Transform (STFT) [2] is an interesting solution, but is however limited by its poor energy localization in the time-frequency (TF) plane [3]-[5]. One approach, reassignment [6]-[8], provides an improved time-frequency representation (TFR), but is unfortunately not invertible. This is a reason why synchrosqueezing was introduced, to improve the TF localization of a TFR by reassigning its value rather than its squared modulus [9], [10]. While maintaining invertibility, synchrosqueezing allows TF analysis, denoising or mode extraction [11]-[13]. To improve the localization of strongly modulated signals, a so-called second-order synchrosqueezed STFT was recently proposed in [14], [15], based on an enhanced instantaneous frequency estimator. All these TFRs use operators derived from additional STFTs using particular windows. Such operators can also be defined to estimate local characteristic parameters of a signal. This letter investigates the local estimation of the chirp rate (CR) and of the instantaneous frequency (IF) of multicomponent signals. It also proposes an unbiased second-order synchrosqueezed STFT that can be implemented using causal recursive filters [1]. Our contributions are threefold.

- We show properties of a non-stationary signal model STFT (Section II) which are related to existing local Chirp Rate Estimators (CREs) and we introduce several new ones, based on a less restrictive signal model, whatever the analysis window.

- We present instantaneous frequency estimators (IFEs) (Section IV) and use them to derive new versions of the synchrosqueezed STFT. For this, we use an analysis window allowing a causal recursive implementation designed for a real-time computation.

This research was supported by the French ANR ASTRES project (ANR13-BS03-0002-01).
- The accuracy of both new CREs and IFEs is comparatively assessed by numerical simulations (Section VI).

\section{SignAl MODEL AND ITS STFT}

\section{A. Signal model and properties}

At every moment, the analyzed signal is supposed to be locally approximated by a Gaussian-modulated linear chirp $x(t)=A_{x}(t) \mathbf{e}^{j \phi_{x}(t)}$, with $A_{x}(t)=\mathcal{A}_{x} \mathbf{e}^{-\left(t-t_{x}\right)^{2} /\left(2 T_{x}^{2}\right)}$, $\phi_{x}(t)=\varphi_{x}+\omega_{x} t+\alpha_{x} t^{2} / 2$ and $j^{2}=-1$. By definition, $x(t)$ is differentiable and its derivative can be expressed as

$$
\begin{aligned}
\frac{\mathrm{d} x}{\mathrm{~d} t}(t) & =\left(\frac{\mathrm{d}}{\mathrm{d} t}\left(\ln \left(A_{x}(t)\right)\right)+j \frac{\mathrm{d} \phi_{x}}{\mathrm{~d} t}(t)\right) x(t) \\
& =\left(q_{x} t+p_{x}\right) x(t),
\end{aligned}
$$

with $q_{x}=-1 / T_{x}^{2}+j \alpha_{x}$ and $p_{x}=t_{x} / T_{x}^{2}+j \omega_{x}$. The term $q_{x} t+p_{x}=-\left(t-t_{x}\right) / T_{x}^{2}+j\left(\alpha_{x} t+\omega_{x}\right)$ is sometimes referred to as the instantaneous complex frequency [16], [17], and its imaginary part is the signal instantaneous frequency.

\section{B. Signal STFT and properties}

Let $F_{x}^{h}(t, \omega)$ denote the STFT of $x(t)$ using a differentiable analysis window $h(t)$, defined as

$$
\begin{aligned}
F_{x}^{h}(t, \omega) & =\int_{\mathbb{R}} x(u) h(t-u)^{*} \mathbf{e}^{-j \omega u} \mathrm{~d} u \\
& =\mathbf{e}^{-j \omega t} \int_{\mathbb{R}} x(t-u) h(u)^{*} \mathbf{e}^{j \omega u} \mathrm{~d} u
\end{aligned}
$$

where $z^{*}$ is the complex conjugate of $z$. Its partial derivative with respect to time can be written as

$$
\begin{aligned}
& \frac{\partial F_{x}^{h}}{\partial t}(t, \omega)=\int_{\mathbb{R}} x(u) \frac{\mathrm{d} h}{\mathrm{~d} t}(t-u)^{*} \mathbf{e}^{-j \omega u} \mathrm{~d} u \\
& =-j \omega F_{x}^{h}(t, \omega)+\mathbf{e}^{-j \omega t} \int_{\mathbb{R}} \frac{\mathrm{d} x}{\mathrm{~d} t}(t-u) h(u)^{*} \mathbf{e}^{j \omega u} \mathrm{~d} u
\end{aligned}
$$

Replacing $\frac{\mathrm{d} x}{\mathrm{~d} t}(t-u)$ by $\left(q_{x}(t-u)+p_{x}\right) x(t-u)$ then leads to $F_{x}^{\mathcal{D} h}(t, \omega)=-q_{x} F_{x}^{\mathcal{T} h}(t, \omega)+\left(q_{x} t+p_{x}-j \omega\right) F_{x}^{h}(t, \omega)$, (7) where $F_{x}^{\mathcal{D} h}(t, \omega)$ and $F_{x}^{\mathcal{T} h}(t, \omega)$ are two STFTs using $\mathcal{D} h(t)=$ $\frac{\mathrm{d} h}{\mathrm{~d} t}(t)$ and $\mathcal{T} h(t)=t h(t)$ as analysis windows. Differentiating again with respect to $t$ leads to

$$
F_{x}^{\mathcal{D}^{2} h}(t, \omega)=-q_{x} F_{x}^{\mathcal{T} \mathcal{D} h}(t, \omega)+\left(q_{x} t+p_{x}-j \omega\right) F_{x}^{\mathcal{D} h}(t, \omega),
$$

and more generally, for $n \geq 1$,

$$
\begin{aligned}
& \frac{\partial^{n} F_{x}^{h}}{\partial t^{n}}(t, \omega)=F_{x}^{\mathcal{D}^{n} h}(t, \omega)= \\
& -q_{x} F_{x}^{\mathcal{T D}^{n-1} h}(t, \omega)+\left(q_{x} t+p_{x}-j \omega\right) F_{x}^{\mathcal{D}^{n-1} h}(t, \omega),
\end{aligned}
$$

with $\mathcal{D}^{n} h(t)=\frac{\mathrm{d}^{n} h}{\mathrm{~d} t^{n}}(t)$ and $\mathcal{T} \mathcal{D}^{n} h(t)=t \frac{d^{n} h}{d t^{n}}(t)$. 


\section{ChIRP RATE ESTIMATION}

\section{A. Estimator using one partial derivative}

Based on Eq. (7), CREs can first be derived, thus continuing a research theme pioneered by Nelson [18]. If we assume that the amplitude is constant (i.e. if $T_{x}$ goes to infinity), then $p_{x}=j \omega_{x}$ and $q_{x}=j \alpha_{x}$, and Eq. (7) leads to

$$
F_{x}^{\mathcal{D} h}(t, \omega)=-j \alpha_{x} F_{x}^{\mathcal{T} h}(t, \omega)+j\left(\alpha_{x} t+\omega_{x}-\omega\right) F_{x}^{h}(t, \omega) .
$$

Multiplying then by $F_{x}^{h}(t, \omega)^{*}$ and taking the real part leads to a chirp rate estimator based on a first-order derivative of the STFT (provided that the denominator is nonzero),

$$
\hat{\alpha}_{x}^{K 1}(t, \omega)=\frac{\operatorname{Re}\left(F_{x}^{\mathcal{D} h}(t, \omega) F_{x}^{h}(t, \omega)^{*}\right)}{\operatorname{Im}\left(F_{x}^{\mathcal{T} h}(t, \omega) F_{x}^{h}(t, \omega)^{*}\right)} .
$$

This equation, which was first derived in [19], [20] from analytical results obtained in the particular case of a Gaussian window, provides a CRE for any analysis window $h$ (unbiased if $T_{x}^{2} \rightarrow \infty$ ). Based on Eq. (9), a whole class of CREs can also be derived on the same principle:

$$
\hat{\alpha}_{x}^{K n}(t, \omega)=\frac{\operatorname{Re}\left(F_{x}^{\mathcal{D}^{n} h}(t, \omega) F_{x}^{\mathcal{D}^{n-1} h}(t, \omega)^{*}\right)}{\operatorname{Im}\left(F_{x}^{\mathcal{T} \mathcal{D}^{n-1} h}(t, \omega) F_{x}^{\mathcal{D}^{n-1} h}(t, \omega)^{*}\right)} .
$$

\section{B. Estimators using two partial derivatives}

1) Estimators using derivatives with respect to time: If the amplitude is no longer assumed to be constant, Eqs. (7) and (8) can be considered as a system of two linear equations with two variables $q_{x} t+p_{x}-j \omega$ and $q_{x}$. The solution of this system leads to an estimator of $q_{x}$ (provided that the denominator is nonzero), and its imaginary part to a new CRE

$$
\begin{aligned}
\hat{q}_{x}^{(t 2)}(t, \omega) & =\frac{F_{x}^{\mathcal{D}^{2} h}(t, \omega) F_{x}^{h}(t, \omega)-F_{x}^{\mathcal{D} h}(t, \omega)}{F_{x}^{\mathcal{D} h}(t, \omega) F_{x}^{\mathcal{T} h}(t, \omega)-F_{x}^{\mathcal{T} \mathcal{D} h}(t, \omega) F_{x}^{h}(t, \omega)}, \\
\hat{\alpha}_{x}^{(t 2)}(t, \omega) & =\operatorname{Im}\left(\hat{q}_{x}^{(t 2)}(t, \omega)\right) .
\end{aligned}
$$

More generally, a whole class of CREs can be derived if the same process is applied to Eqs. (7) and (9):

$$
\begin{aligned}
\hat{q}_{x}^{(t n)}(t, \omega) & =\frac{F_{x}^{\mathcal{D}^{n} h}(t, \omega) F_{x}^{h}(t, \omega)-F_{x}^{\mathcal{D}^{n-1} h}(t, \omega) F_{x}^{\mathcal{D} h}(t, \omega)}{F_{x}^{\mathcal{D}^{n-1} h}(t, \omega) F_{x}^{\mathcal{T} h}(t, \omega)-F_{x}^{\mathcal{T D}^{n-1} h}(t, \omega) F_{x}^{h}(t, \omega)}, \\
\hat{\alpha}_{x}^{(t n)}(t, \omega) & =\operatorname{Im}\left(\hat{q}_{x}^{(t n)}(t, \omega)\right) .
\end{aligned}
$$

2) Estimators using derivatives with respect to frequency: Since $\frac{\partial F_{x}^{h}}{\partial \omega}=j\left(F_{x}^{\mathcal{T} h}(t, \omega)-t F_{x}^{h}(t, \omega)\right)$, differentiating Eq. (7) once with respect to $\omega$ leads to

$$
\begin{aligned}
& F_{x}^{\mathcal{T} \mathcal{D} h}(t, \omega)+F_{x}^{h}(t, \omega)= \\
& -q_{x} F_{x}^{\mathcal{T}^{2} h}(t, \omega)+\left(q_{x} t+p_{x}-j \omega\right) F_{x}^{\mathcal{T} h}(t, \omega) .
\end{aligned}
$$

More generally, differentiating Eq. (7) $n-1$ times (for $n \geq 2$ ) with respect to $\omega$ leads to (with $\mathcal{T}^{n} h(t)=t^{n} h(t)$ )

$$
\begin{aligned}
& F_{x}^{\mathcal{T}^{n-1} \mathcal{D} h}(t, \omega)+(n-1) F_{x}^{\mathcal{T}^{n-2} h}(t, \omega)= \\
& -q_{x} F_{x}^{\mathcal{T}^{n} h}(t, \omega)+\left(q_{x} t+p_{x}-j \omega\right) F_{x}^{\mathcal{T}^{n-1} h}(t, \omega) .
\end{aligned}
$$

Considering Eqs. (7) and (15) as a set of two linear equations leads to another estimator of $q_{x}$ and to another CRE

$$
\begin{aligned}
& \hat{q}_{x}^{(\omega 2)}=\frac{F_{x}^{\mathcal{T} \mathcal{D} h}(t, \omega) F_{x}^{h}(t, \omega)+F_{x}^{h}(t, \omega)^{2}-F_{x}^{\mathcal{T} h}(t, \omega) F_{x}^{\mathcal{D} h}(t, \omega)}{F_{x}^{\mathcal{T} h}(t, \omega)^{2}-F_{x}^{\mathcal{T}^{2} h}(t, \omega) F_{x}^{h}(t, \omega)}, \\
& \hat{\alpha}_{x}^{(\omega 2)}=\operatorname{Im}\left(\hat{q}_{x}^{(\omega 2)}(t, \omega)\right) .
\end{aligned}
$$

More generally, a whole class of CREs can be derived from Eqs. (7) and (16):

$$
\begin{aligned}
& \hat{q}_{x}^{(\omega n)}=\frac{\left(F_{x}^{\mathcal{T}^{n-1} \mathcal{D} h}+(n-1) F_{x}^{\mathcal{T}^{n-2} h}\right) F_{x}^{h}-F_{x}^{\mathcal{T}^{n-1} h} F_{x}^{\mathcal{D} h}}{F_{x}^{\mathcal{T}^{n-1} h} F_{x}^{\mathcal{T} h}-F_{x}^{\mathcal{T}^{n} h} F_{x}^{h}}, \\
& \hat{\alpha}_{x}^{(\omega n)}=\operatorname{Im}\left(\hat{q}_{x}^{(\omega n)}(t, \omega)\right) .
\end{aligned}
$$

Of course, the dependency of these estimators and STFTs to the TF point $(t, \omega)$ has been removed by lack of space, and these expressions hold when their denominators are non zero.

\section{INSTANTANEOUS FREQUENCY ESTIMATION}

\section{A. Bias of the frequency reassignment operator}

Classical spectrogram reassignment and synchrosqueezing use time and frequency reassignment operators defined as [1], [6]-[8], [21]

$$
\begin{aligned}
\hat{t}(t, \omega) & =\operatorname{Re}(\tilde{t}(t, \omega)), \text { with } \quad \tilde{t}(t, \omega)=t-\frac{F_{x}^{\mathcal{T} h}(t, \omega)}{F_{x}^{h}(t, \omega)}, \\
\hat{\omega}(t, \omega) & =\operatorname{Im}(\tilde{\omega}(t, \omega)), \text { with } \quad \tilde{\omega}(t, \omega)=j \omega+\frac{F_{x}^{\mathcal{D} h}(t, \omega)}{F_{x}^{h}(t, \omega)} .
\end{aligned}
$$

Eq. (7) shows that when the signal amplitude is not constant, i.e. when $T_{x}^{2}<+\infty, \hat{\omega}(t, \omega)$ provides a biased IF estimation at time $\hat{t}(t, \omega)$, since Eqs. (7), (19) and (20) lead to

$$
\hat{\omega}(t, \omega)=\alpha_{x} \hat{t}(t, \omega)+\omega_{x}+\frac{1}{T_{x}^{2}} \operatorname{Im}\left(\frac{F_{x}^{\mathcal{T} h}(t, \omega)}{F_{x}^{h}(t, \omega)}\right) .
$$

\section{B. Unbiased instantaneous frequency estimators}

Unbiased estimators of the instantaneous frequency at time $t, \dot{\phi}_{x}(t)=\alpha_{x} t+\omega_{x}$, can be derived from the results presented in Section III. When the signal amplitude is constant, Eq. (21) shows that $\dot{\phi}_{x}(t, \omega)=\hat{\omega}+\hat{\alpha}_{x}^{K 1}(t-\hat{t})$ is an unbiased IFE. When the signal amplitude is a Gaussian, Eqs. (7), (19) and (20) lead to the simple relationship $\tilde{\omega}(t, \omega)=q_{x} \tilde{t}(t, \omega)+p_{x}$, from which IFEs can be deduced by [22] $\hat{\dot{\phi}}_{x}(t, \omega)=\operatorname{Im}\left(\tilde{\omega}+\hat{q}_{x}(t-\tilde{t})\right)$. If $\hat{q}_{x}$ is given by Eqs. (13), (14), (17) or (18), these IFEs are unbiased, whatever the analysis window $h$.

Furthermore, unbiased IFEs can also be derived directly, for a lower computational cost and a lower memory requirement. When the signal amplitude is constant, multiplying Eq. (10) by $F_{x}^{\mathcal{T} h}(t, \omega)^{*}$ and taking the real part leads to

$$
\hat{\dot{\phi}}_{x}^{K 1}(t, \omega)=\omega-\frac{\operatorname{Re}\left(F_{x}^{\mathcal{D} h}(t, \omega) F_{x}^{\mathcal{T} h}(t, \omega)^{*}\right)}{\operatorname{Im}\left(F_{x}^{h}(t, \omega) F_{x}^{\mathcal{T} h}(t, \omega)^{*}\right)} .
$$

Compared to Eq. (20), $\hat{\dot{\phi}}_{x}^{K 1}(t, \omega)$ is an estimator of the IF at time $t$, whereas $\hat{\omega}(t, \omega)$ is an estimator of the IF at time $\hat{t}(t, \omega)$, $\dot{\phi}_{x}(\hat{t}(t, \omega), \omega)$. On the same principle, a whole class of IFEs can be derived from Eq. (9),

$$
\hat{\dot{\phi}}_{x}^{K n}(t, \omega)=\omega-\frac{\operatorname{Re}\left(F_{x}^{\mathcal{D}^{n} h}(t, \omega) F_{x}^{\mathcal{T} \mathcal{D}^{n-1} h}(t, \omega)^{*}\right)}{\operatorname{Im}\left(F_{x}^{\mathcal{D}^{n-1} h}(t, \omega) F_{x}^{\mathcal{T} \mathcal{D}^{n-1} h}(t, \omega)^{*}\right)} .
$$

For a linearly frequency modulated signal with an amplitude locally approximated by a Gaussian, unbiased IFEs can also be derived. Combining Eqs. (7) and (8) leads to an estimator of $q_{x} t+p_{x}-j \omega$, whose imaginary part yields an IFE and 
which can be generalized to any order from Eqs. (7) and (16) (provided that the denominators are nonzero)

$$
\begin{aligned}
& \hat{\dot{\phi}}_{x}^{(t 2)}(t, \omega)=\omega+\operatorname{Im}\left(\frac{F_{x}^{\mathcal{D}^{2} h} F_{x}^{\mathcal{T} h}-F_{x}^{\mathcal{T} \mathcal{D} h} F_{x}^{\mathcal{D} h}}{F_{x}^{\mathcal{D} h} F_{x}^{\mathcal{T} h}-F_{x}^{\mathcal{T} \mathcal{D} h} F_{x}^{h}}\right), \\
& \hat{\dot{\phi}}_{x}^{(t n)}(t, \omega)=\omega+\operatorname{Im}\left(\frac{F_{x}^{\mathcal{D}^{n} h} F_{x}^{\mathcal{T} h}-F_{x}^{\mathcal{T} \mathcal{D}^{n-1} h} F_{x}^{\mathcal{D} h}}{F_{x}^{\mathcal{D}^{n-1} h} F_{x}^{\mathcal{T} h}-F_{x}^{\mathcal{T} \mathcal{D}^{n-1} h} F_{x}^{h}}\right) .
\end{aligned}
$$

Combining Eqs. (7) and (16) can also lead to another class of instantaneous frequency estimators, defined as $\hat{\dot{\phi}}_{x}^{(\omega n)}(t, \omega)=$

$$
\omega+\operatorname{Im}\left(\frac{\left(F_{x}^{\mathcal{T}^{n-1} \mathcal{D} h}+(n-1) F_{x}^{\mathcal{T}^{n-2} h}\right) F_{x}^{\mathcal{T} h}-F_{x}^{\mathcal{T}^{n} h} F_{x}^{\mathcal{D} h}}{F_{x}^{\mathcal{T}^{n-1} h} F_{x}^{\mathcal{T} h}-F_{x}^{\mathcal{T}^{n} h} F_{x}^{h}}\right) .
$$

\section{Vertical synchrosqueezing}

The results presented in Section IV-B can be used to estimate the IF of a signal component located in the vicinity of a TF point $(t, \omega)$. It can also be used to derive an improved synchrosqueezing process called vertical synchrosqueezing: based on the general signal reconstruction formula [1]

$$
x\left(t-t_{0}\right)=\frac{1}{h\left(t_{0}\right)^{*}} \int_{\mathbb{R}} F_{x}^{h}(t, \omega) \mathbf{e}^{j \omega\left(t-t_{0}\right)} \frac{\mathrm{d} \omega}{2 \pi},
$$

for any $t_{0}$ such that $h\left(t_{0}\right) \neq 0$, a vertically synchrosqueezed STFT can be defined as [15], [22]

$$
V S F_{x}^{h}(t, \omega)=\int_{\mathbb{R}} F_{x}^{h}\left(t, \omega^{\prime}\right) \mathbf{e}^{j \omega^{\prime}\left(t-t_{0}\right)} \delta\left(\omega-\hat{\dot{\phi}}_{x}\left(t, \omega^{\prime}\right)\right) \mathrm{d} \omega^{\prime},
$$

where $\hat{\dot{\phi}}_{x}(t, \omega)$ is one of the proposed IFEs. This expression mainly differs from the classical synchrosqueezed STFT by the use of an IFE $\hat{\dot{\phi}}_{x}\left(t, \omega^{\prime}\right)$ instead of $\hat{\omega}\left(t, \omega^{\prime}\right)$. Its squared modulus provides a sharpened TFR, and it can be inverted by

$$
\hat{x}\left(t-t_{0}\right)=\frac{1}{h\left(t_{0}\right)^{*}} \int_{\mathbb{R}} V S F_{x}^{h}(t, \omega) \frac{\mathrm{d} \omega}{2 \pi},
$$

where the integration interval can profitably be restricted to the vicinity of a signal ridge for mode extraction [13].

\section{ROBUSTNESS ANALYSIS AND ROBUST CR AND IF ESTIMATORS}

All the previously proposed estimators are deduced from STFT properties induced by the chosen signal model. To assess their sensitivity to a model inadequacy, let's suppose that the log-amplitude and the phase are third-order polynomials:

$$
\begin{aligned}
\ln \left(A_{x}(t)\right) & =\ln \left(\mathcal{A}_{x}\right)-\frac{\left(t-t_{x}\right)^{2}}{2 T_{x}^{2}}-\Delta_{A} \frac{\left(t-t_{x}\right)^{3}}{6 T_{x}^{2}}, \\
\phi_{x}(t) & =\varphi_{x}+\omega_{x} t+\alpha_{x} t^{2} / 2+\Delta_{\phi} t^{3} / 6 .
\end{aligned}
$$

Eqs. (2) and (7) then become $\frac{\mathrm{d} x}{\mathrm{~d} t}(t)=\left(r_{x} t^{2}+q_{x} t+p_{x}\right) x(t)$, with $r_{x}=-\frac{\Delta_{A}}{2 T_{x}^{2}}+j \frac{\Delta_{\phi}}{2}, q_{x}=-\frac{1-\Delta_{A} t_{x}}{T_{x}^{2}}+j \alpha_{x}$ and $p_{x}=$ $\frac{2 t_{x}-\Delta_{A} t_{x}^{2}}{2 T_{x}^{2}}+j \omega_{x}$, and $F_{x}^{\mathcal{D} h}(t, \omega)=r_{x} F_{x}^{\mathcal{T}^{2} h}(t, \omega)-$

$$
\left(2 r_{x} t+q_{x}\right) F_{x}^{\mathcal{T} h}(t, \omega)+\left(r_{x} t^{2}+q_{x} t+p_{x}-j \omega\right) F_{x}^{h}(t, \omega) .
$$

This expression mainly differs from Eq (7) by an additional term proportional to $r_{x}$, and shows that Eqs. (11) and (22) are biased estimators of the instantaneous chirp rate $\Delta_{\phi} t+\alpha_{x}=$
$\operatorname{Im}\left(2 r_{x} t+q_{x}\right)$ and of the instantaneous frequency $\Delta_{\phi} t^{2} / 2+$ $\alpha_{x} t+\omega_{x}=\operatorname{Im}\left(r_{x} t^{2}+q_{x} t+p_{x}\right)$, and the bias is proportional to $\Delta_{\phi}$. Differentiating Eq. (32) once with respect to $t$ and with respect to $\omega$ leads to

$$
\begin{aligned}
F_{x}^{\mathcal{D}^{2} h} & =r_{x} F_{x}^{\mathcal{T}^{2} \mathcal{D} h}-\left(2 r_{x} t+q_{x}\right) F_{x}^{\mathcal{T} \mathcal{D} h} \\
& +\left(r_{x} t^{2}+q_{x} t+p_{x}-j \omega\right) F_{x}^{\mathcal{D} h} \\
F_{x}^{\mathcal{T} \mathcal{D} h}+F_{x}^{h} & =r_{x} F_{x}^{\mathcal{T}^{3} h}-\left(2 r_{x} t+q_{x}\right) F_{x}^{\mathcal{T}^{2} h} \\
& +\left(r_{x} t^{2}+q_{x} t+p_{x}-j \omega\right) F_{x}^{\mathcal{T} h} .
\end{aligned}
$$

These expressions show that Eqs. (13), (17), (24) and (26) (for $n=2$ ) are biased estimators of the instantaneous chirp rate and of the instantaneous frequency, with a bias proportional to $\Delta_{\phi}$ and independent of $\Delta_{A}$. However, Eqs. (32), (33) and (34) can be considered as a set of three linear equations with three variables $r_{x}, 2 r_{x} t+q_{x}$ and $r_{x} t^{2}+q_{x} t+p_{x}-j \omega$. When the determinant is nonzero, the imaginary parts of the solutions of this system can lead to robust estimators of the angular jerk $\Delta_{\phi}$, of the instantaneous chirp rate and of the instantaneous frequency.

\section{NUMERICAL RESULTS}

The results presented in the previous sections hold for any analysis window $h$. We implemented them for causal (onesided) analysis windows $h_{k}$ (with $k \geq 1$ ) defined by [1], [24] $h_{k}(t)=\frac{t^{k-1}}{T^{k}(k-1) !} \mathbf{e}^{-t / T} U(t)$, where $U(t)$ is the Heaviside step function and $T$ a time spread parameter. This allows an efficient recursive discrete-time implementation of the STFT and of the CR and IF estimators, since $\mathcal{T}^{n} h$ and $\mathcal{D}^{n} h$ can be expressed as linear combinations of functions of this kind [1]. A MATLAB implementation of the proposed estimators and a complementary report can be found on-line at [25].

\section{A. Statistical efficiency assessment}

We considered a complex linear chirp $x[n]=A_{x}[n] \mathbf{e}^{j \alpha \frac{n^{2}}{2}}$ (with $A_{x}[n]=\mathrm{e}^{-\left(n-n_{0}\right)^{2} /\left(2 L_{x}^{2}\right)}, n \in[0, N-1], n_{0}=N / 2$, $\alpha=2 \pi \frac{0.36}{N}, N=500$ and $L_{x}=200$ or $\left.L_{x}=+\infty\right)$, merged in a circular white Gaussian noise with a Signal-to-Noise Ratio (SNR) varying from -10 to $+80 \mathrm{~dB}$. The CR and IF are estimated at $n_{0}=N / 2$ and the error is measured from the ground-truth values $\alpha_{\text {ref }}=4.5 \cdot 10^{-3}$ and $\lambda_{\text {ref }}=\frac{\alpha_{\text {ref }}}{2 \pi} n_{0}=0.18$. Figs. 1(a) and 1(c) show the Mean Squared Error (MSE) of the CR and IF estimators computed at $n_{0}$ with 50 noise realizations, as a function of the analysis frequency $\lambda=\frac{\omega}{2 \pi T_{s}}$ ( $T_{s}$ being the sampling period). These results show that most estimators best perform in the vicinity of the maximum of the spectrogram, except for the K1 estimators, where a division by zero then happens (as previously reported in [19], [20]). The latter estimators also become biased when the signal amplitude is not constant. This is confirmed by Figs. 1(b) and 1(d), which compare the MSE of the estimators as a function of the SNR. In these figures, the MSE also consider 36 distinct uniformly sampled values for $\lambda \in[0.13,0.20]$ around the maximum of the spectrogram (i.e. 1800 measures per point). The results show that K1 estimators are less efficient for low SNR. At high SNR, the MSE of the proposed estimators seems to be inversely proportional to the SNR, except for the K1 estimators when the signal amplitude is Gaussian $\left(L_{x}=200\right)$. 


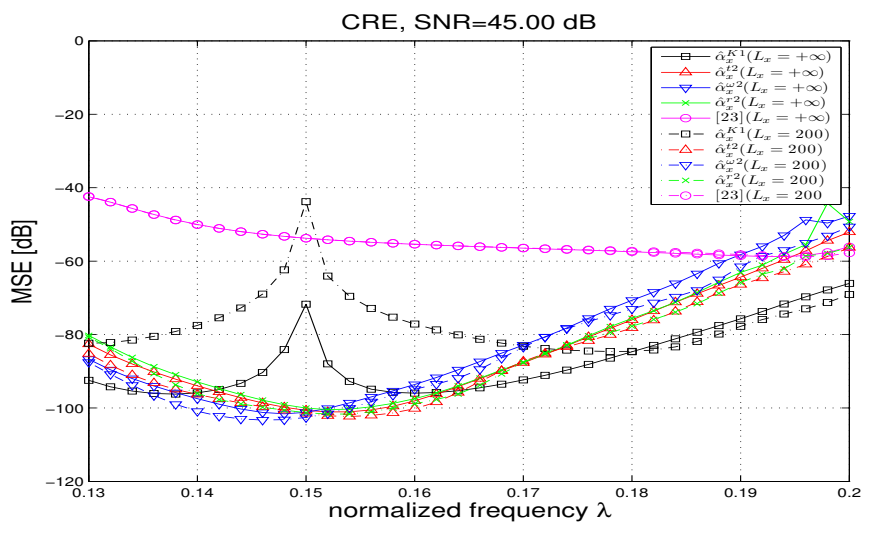

(a) Mean Squared Error of the CREs

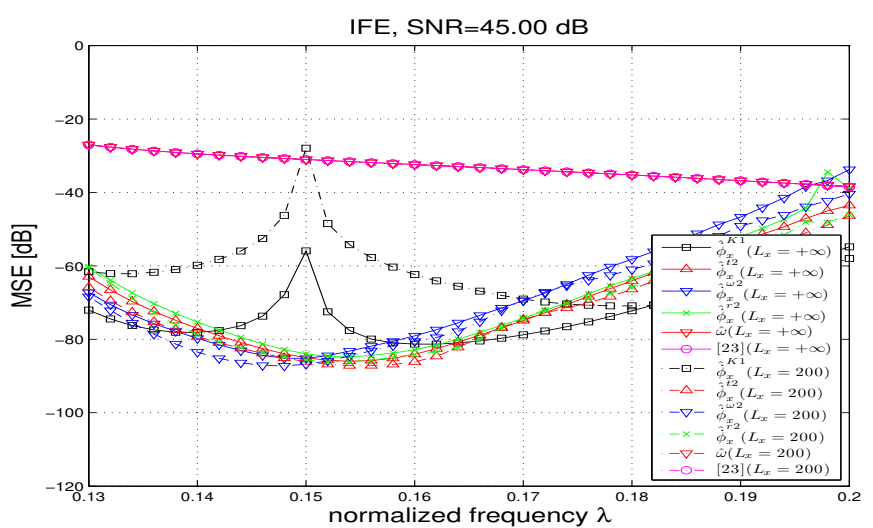

(c) Mean Squared Error of the IFEs

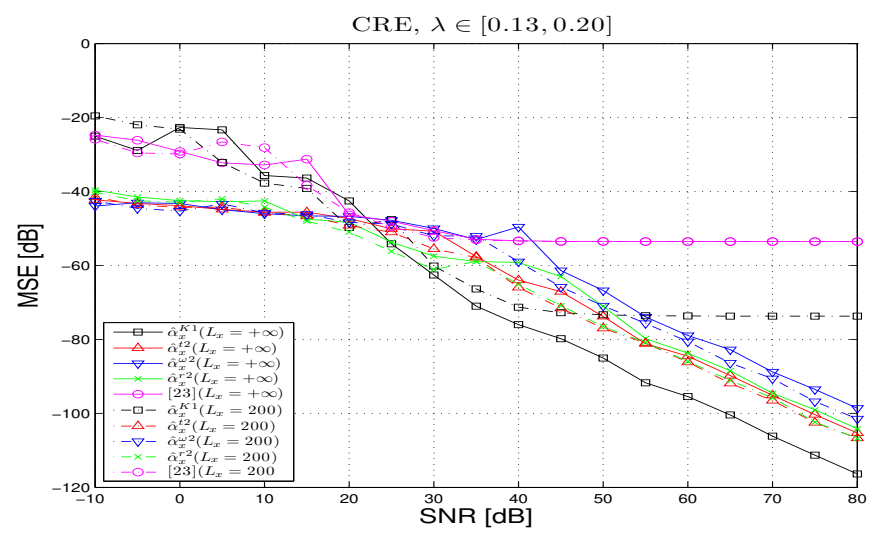

(b) CRE robustness to noise

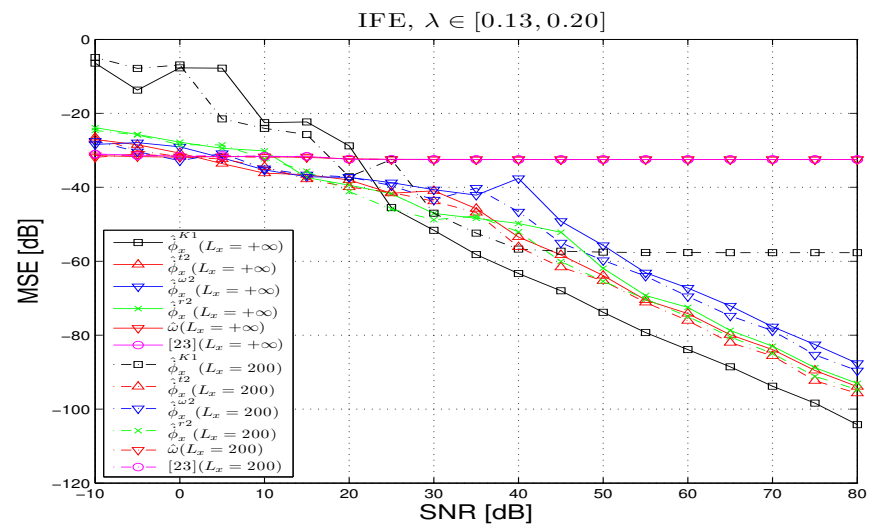

(d) IF estimation robustness to noise

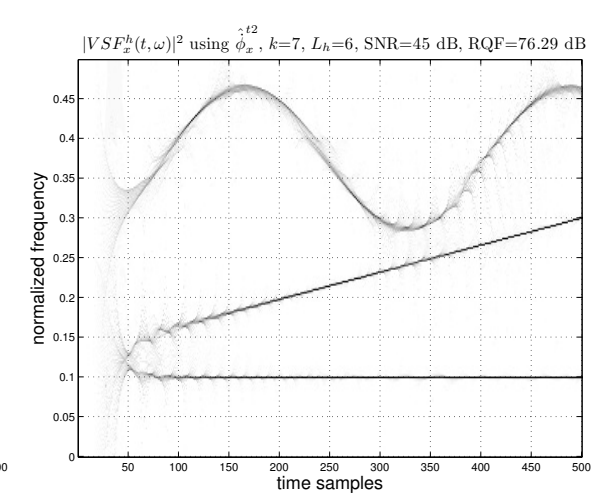

(g) $\left|V S F_{x}^{h}(t, \omega)\right|^{2}$ using Eq. (24)

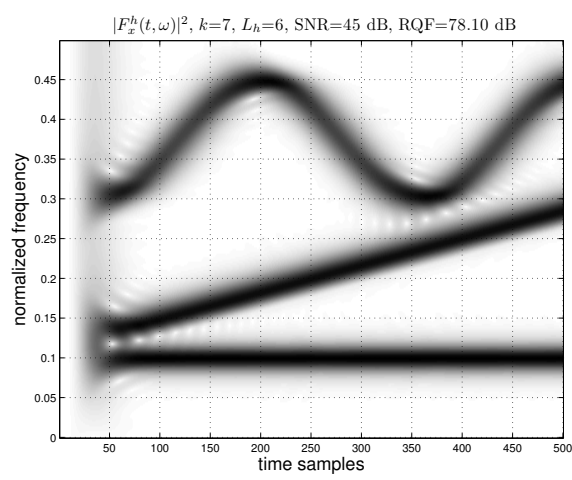

(e) spectrogram

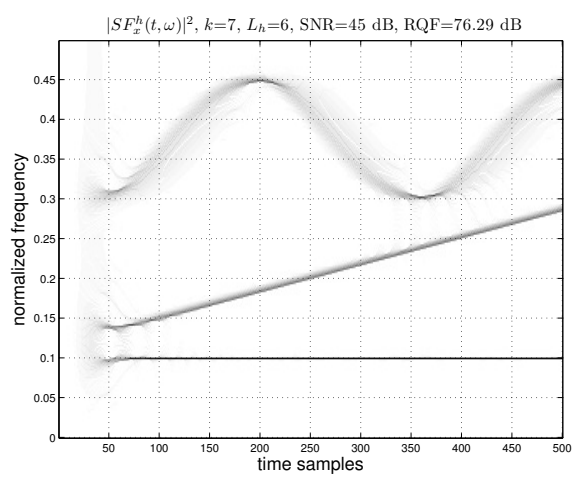

(f) classical synchrosqueezing

Fig. 1. Accuracy assessment of the proposed CREs (a)-(b) and IFEs (c)-(d), in comparison with a state-of-the-art method [23], applied to a constant amplitude linear chirp. Recursive TFRs of a multicomponent signal (e)-(g). For all figures, the order is $k=7$ and the time spread is $L_{h}=T / T_{s}=6$ (see [1]).

\section{B. Time-frequency localization assessment}

Figs. 1 (e)-(g) compare the recursive TFRs obtained for a real signal made of one sinusoid, one chirp and one sinusoidally modulated sinusoid. As shown in [1], synchrosqueezing (Fig. 1(f)) improves the localization of the signal components with some noise robustness. However, a significant TFR improvement (clearly visible on the two frequency modulated components) is provided using Eq. (24) (Fig. 1(g)) instead of Eq. (20) (Fig. 1(f)). All these TFRs remain invertible, as shown by the Reconstruction Quality Factor (RQF) [1] displayed above each figure. Hence, the better TF localization provided by the proposed recursive vertical synchrosqueezing could improve mode retrieval as in [13], with a more efficient computation.

\section{CONCLUSION}

This paper illustrates the possibility to measure at any TF point not only energy, but also signal parameters, even when using one-sided windows that allow a real-time recursive implementation. Based on a local signal model, general expressions for any analysis window, allow us to derive and investigate an infinity of new unbiased CR and IF estimators, valid whatever the analysis window. Similarly, estimators of the signal parameters $t_{x}$ and $T_{x}$ could also be designed. Future research includes the use of other signal models (such as hyperbolic chirps), a study of the poles of the proposed estimators, a statistical assessment of the high-order $(n>2)$ CR and IF estimators, and applications to real-world signals. 


\section{REFERENCES}

[1] D. Fourer, F. Auger, and P. Flandrin, "Recursive versions of the Levenberg-Marquardt reassigned spectrogram and of the synchrosqueezed STFT," in Proc. IEEE ICASSP, Mar. 2016, pp. 4880-4884.

[2] J. Allen, "Short term spectral analysis, synthesis, and modification by discrete Fourier transform," IEEE Trans. Acoust., Speech, Signal Process., vol. 25, no. 3, pp. 235-238, Jun. 1977.

[3] P. Flandrin, Time-Frequency/Time-Scale analysis. Acad. Press, 1998.

[4] L. Cohen, Time-Frequency Analysis: Theory and Applications. Prentice Hall, 1995.

[5] F. Hlawatsch and F. Auger, Eds., Time-Frequency Analysis: Concepts and Methods. ISTE-Wiley, 2008.

[6] K. Kodera, C. de Villedary, and R. Gendrin, "A new method for the numerical analysis of non-stationary signals," Physics of the Earth and Planetary Interiors, vol. 12, pp. 142-150, 1976.

[7] F. Auger and P. Flandrin, "Improving the readability of time-frequency and time-scale representations by the reassignment method," IEEE Trans. Signal Process., vol. 43, no. 5, pp. 1068-1089, May 1995.

[8] F. Auger, E. Chassande-Mottin, and P. Flandrin, "Making reassignment adjustable: the Levenberg-Marquardt approach," in Proc. IEEE ICASSP, Mar. 2012, pp. 3889-3892.

[9] I. Daubechies and S. Maes, "A nonlinear squeezing of the continuous wavelet transform," Wavelets in Medecine and Bio., pp. 527-546, 1996.

[10] T. Oberlin, S. Meignen, and V. Perrier, "The Fourier-based synchrosqueezing transform," in Proc. IEEE ICASSP, 2014, pp. 315-319.

[11] K. Czarnecki and M. Moszyński, "Using concentrated spectrogram for analysis of audio acoustic signals," Hydroacoustics, vol. 15, pp. 27-32, 2012.

[12] K. Czarnecki, D. Fouan, Y. Achaoui, and S. Mensah, "Fast bubble dynamics and sizing," Journal of Sound and Vibration, vol. 356, pp. 48-60, 2015.

[13] S. Meignen, T. Oberlin, and S. McLaughlin, "A new algorithm for multicomponent signals analysis based on synchrosqueezing: with an application to signal sampling and denoising," IEEE Trans. Signal Process., vol. 60, no. 11, pp. 5787-5798, Nov. 2012.

[14] T. Gardner and M. Magnasco, "Sparse time-frequency representations," Proceedings of the National Academy of Sciences, vol. 3, no. 16, pp. 6094-6099, 2006.

[15] T. Oberlin, S. Meignen, and V. Perrier, "Second-order synchrosqueezing transform or invertible reassignment? Towards ideal time-frequency representations," IEEE Trans. Signal Process., vol. 63, no. 5, pp. 13351344, Mar. 2015.

[16] D. A. Linden, "A note concerning instantaneous frequency," Proc. of the IRE, vol. 46, p. 1970, 1958.

[17] S. L. Hahn, "The instantaneous complex frequency concept and its application to the analysis of building-up of oscillations in oscillators," Proceedings of Vibration Problems, vol. 1, pp. 24-46, 1959.

[18] D. Nelson, "Instantaneous higher order phase derivatives," Digital Signal Processing, vol. 12, no. 2-3, pp. 416-428, 2002.

[19] K. Czarnecki and M. Moszyński, "A novel method of local chirp-rate estimation of LFM chirp signals in the time-frequency domain," in 36th Int. Conf. on Telecommunications and Signal Proc., 2013, pp. 704-708.

[20] K. Czarnecki, "The instantaneous frequency rate spectrogram," Mechanical Systems ans Signal Processing, vol. 66-67, pp. 361-373, 2016.

[21] F. Auger, E. Chassande-Mottin, and P. Flandrin, "On phase-magnitude relationships in the short-time Fourier transform," IEEE Signal Process. Lett., vol. 19, no. 5, pp. 267-270, May 2012.

[22] R. Behera, S. Meignen, and T. Oberlin, "Theoretical analysis of the second-order synchrosqueezing transform," hal-01220017, 2015.

[23] S. Marchand, "The simplest analysis method for non-stationary sinusoidal modeling," in Proc. 15th International Conference on Digital Audio Effects (DAFx-12), 2012, pp. 23-26.

[24] G. Nilsen, "Recursive time-frequency reassignment," IEEE Trans. Signal Process., vol. 57, no. 8, pp. 3283-3287, Aug. 2009.

[25] "ASTRES project MATLAB resources," http://www.ens-lyon.fr/ PHYSIQUE/Equipe3/ANR_ASTRES/resources.html. 